\title{
Spatially resolved microwave pulsations of a flare loop
}

\author{
V. F. Melnikov ${ }^{1}$, V. E. Reznikova ${ }^{1}$, K. Shibasaki ${ }^{2}$, and V. M. Nakariakov ${ }^{3}$ \\ 1 Radiophysical Research Institute (NIRFI), Nizhny Novgorod, 603950, Russia \\ e-mail: meln@nirfi.sci-nnov.ru \\ 2 Nobeyama Radio Observatory, National Astronomical Observatory of Japan, Minamimaki, Minamisaku, Nagano 384-1305, \\ Japan \\ 3 Physics Department, University of Warwick, Coventry CV4 7AL, UK
}

Received 26 January 2005 / Accepted 24 April 2005

\begin{abstract}
A microwave burst with quasi-periodic pulsations was studied with high spatial resolution using observations with the Nobeyama Radioheliograph (NoRH). We found that the time profiles of the microwave emission at 17 and $34 \mathrm{GHz}$ exhibit quasi-periodic (with two well defined periods $P_{1}=14-17 \mathrm{~s}$ and $P_{2}=8-11 \mathrm{~s}$ ) variations of the intensity at different parts of an observed flaring loop. Detailed Fourier analysis shows the $P_{1}$ spectral component to be dominant at the top, while the $P_{2}$ one near the feet of the loop. The 14-17 s pulsations are synchronous at the top and in both legs of the loop. The 8-11 s pulsations at the legs are well correlated with each other but the correlation is not so obvious with the pulsations at the loop top. For this $P_{2}$ spectral component, a definite phase shift, $P_{2} / 4 \approx 2.2 \mathrm{~s}$, between pulsations in the northern leg and loop top parts of the loop have been found. The length of the flaring loop is estimated as $L=25 \mathrm{Mm}\left(\approx 34^{\prime \prime}\right)$ and its average width at half intensity at $34 \mathrm{GHz}$ as about $6 \mathrm{Mm}\left(\approx 8^{\prime \prime}\right)$. Microwave diagnostics shows the loop to be filled with a dense plasma with the number density $n_{0} \approx 10^{11} \mathrm{~cm}^{-3}$, penetrated by the magnetic field changing from $B_{0} \approx 100 \mathrm{G}$ near the loop top up to $B_{0} \approx 200 \mathrm{G}$ near the north footpoint. A comparative analysis of different MHD modes of the loop demonstrates the possibility of the simultaneous existence of two modes of oscillations in the loop: the global sausage mode, with the period $P_{1}=14-17 \mathrm{~s}$ and the nodes at the footpoints, and a higher harmonics mode (possibly with the radial wave number $l>1$ ), with $P_{2}=8-11 \mathrm{~s}$.
\end{abstract}

Key words. magnetohydrodynamics (MHD) - Sun: activity - Sun: flares - Sun: oscillations - Sun: radio radiation

\section{Introduction}

Spatially unresolved quasi-periodic pulsations with periods of about $1-20 \mathrm{~s}$ are often detected in the microwave and hard X-ray emission generated during solar flares (Kane et al. 1983; Kiplinger et al. 1983; Nakajima 1983; Urpo et al. 1992). Such a phenomenon is usually interpreted in terms of MHD oscillations (magnetoacoustic or Alfvén) of flaring loops, modulating the gyrosynchrotron emission of trapped nonthermal electrons (Rosenberg 1970), or electron precipitation in the dense layers of the solar atmosphere (Zaitsev \& Stepanov 1982, 1989). The generation of the MHD oscillations is usually associated with either the initial energy release in the dense kernel of the flare (Zaitsev \& Stepanov 1982), or with the plasma flows induced by chromospheric evaporation (Zaitsev \& Stepanov 1989). Alternative mechanisms for the generation of MHD oscillations are the particle acceleration in current sheets (Aschwanden 1987) and in colliding current-carrying loops (Sakai \& de Jager 1996), and in a single current-carrying loop considered as an LCR-circuit (Zaitsev et al. 1998).

On the other hand, high spatial and temporal resolution EUV imagers onboard SOHO and TRACE spacecraft have brought a major breakthrough in the observational study of coronal MHD wave activity, revealing the presence of kink and slow magnetoacoustic oscillations of coronal loops (see Nakariakov 2003). Obviously, this progress was connected with the possibility to resolve the wavelength of the oscillation, the opportunity that has been traditionally neglected in radio observations. However, some radio observational facilities, e.g. NoRH, have quite a reasonable spatial resolution $\left(10^{\prime \prime}\right.$ in $17 \mathrm{GHz}$ and $5^{\prime \prime}$ in $34 \mathrm{GHz}$ ), providing us with a tool for the study of the spatial structure of the oscillations, similarly to the EUV band. This, together with the excellent time resolution, can allow for confident identification of observed MHD modes. First attempts to analyse the microwave pulsations of flaring loops using NoRH images were undertaken by Asai et al. (2001), Melnikov et al. (2002b), Grechnev et al. (2003), Nakariakov et al. (2003). In the latter paper, it was demonstrated that the spatial distribution of an observed oscillation is crucial for mode identification.

In this paper, we present a detailed study of an oscillating loop observed with high spatial resolution in $\mathrm{cm}, \mathrm{mm}$ and HXR bands. We obtained information about the spatial structure of the oscillations and their phases in different parts of the flaring loop. The longitudinal and transverse scales of the microwave flaring loop, the distribution of the brightness temperature and the steepness of the spectral slope in different parts 


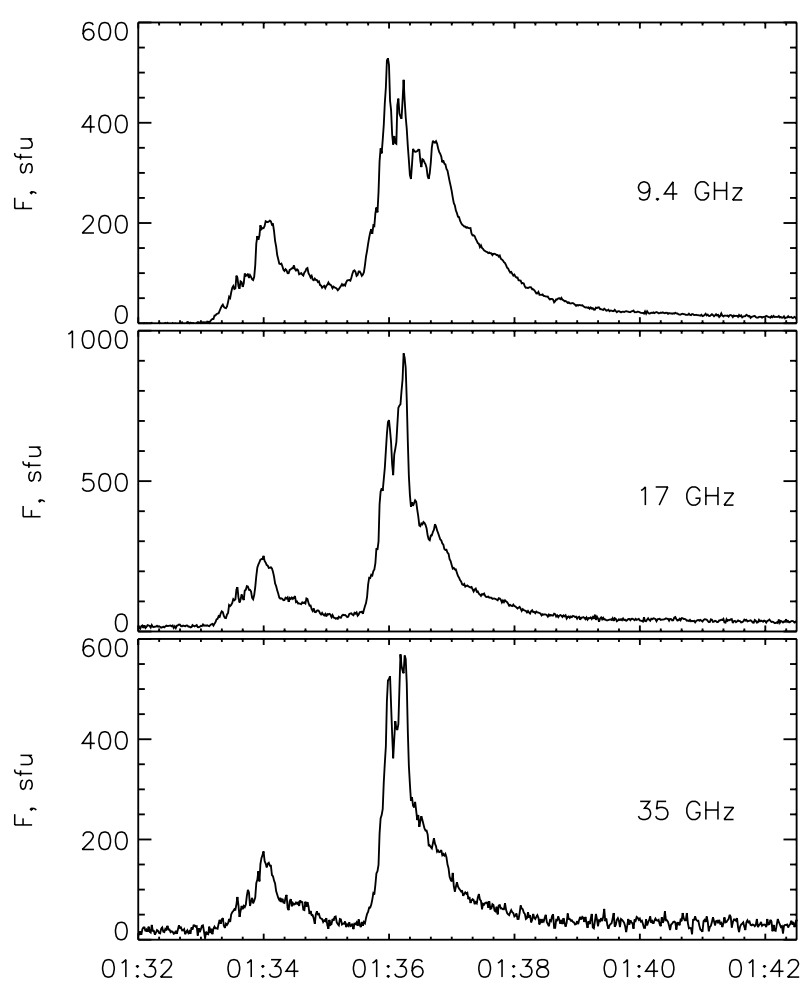

Fig. 1. Total flux time profiles of the event 2000 January 12, obtained by the Nobeyama radiopolarimeter (NoRP) at 9.4, 17, $35 \mathrm{GHz}$.

of the loop are utilised to determine physical conditions inside the loop. The results obtained impose new constraints on the possible MHD oscillation modes in flaring loops and allow us to identify the modes observed.

\section{Observations}

The analysed flare occurred on the 12th of January, 2000, at the Eastern limb (N13E80) and was observed with both the Nobeyama Radioheliograph (NoRH) and the Radiopolarimeter (NoRP). Also, the flare was detected with SXT, HXT and WBS instruments onboard Yohkoh.

\subsection{Total emission fluxes}

Time profiles of the total radio emission of the flare, recorded by NoRP at 9.4, 17 and $35 \mathrm{GHz}$ are shown in Fig. 1. Figure 2 shows the frequency spectrum of the emission during the maximum of the flare microwave burst at 01:36:13 UT, constructed with the NoRP radio fluxes. The peak frequency is in the $17-35 \mathrm{GHz}$ band, with the most probable value at about $25 \mathrm{GHz}$.

The spectral analysis of the NoRP burst light curves reveals $8-15$ s oscillations superposed on the smooth variations of the microwave flux. During the main (second) peak the mean amplitude of the observed quasi-periodical pulsations is about 100 s.f.u.

The existence of the pulsations is confirmed by more sensitive observations with NoRH. Figures 3a,c show the average correlation amplitude $\rho(t)$ of the signals recorded by NoRH at 17 and $34 \mathrm{GHz}$, obtained by averaging the amplitude of

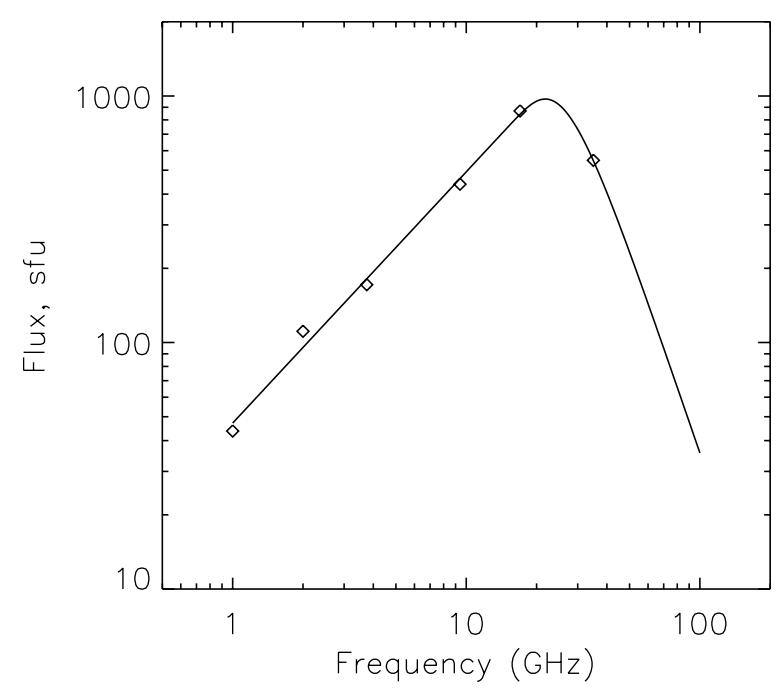

Fig. 2. The NoRP frequency spectrum at the time of the microwave burst peak 01:36:13 UT.

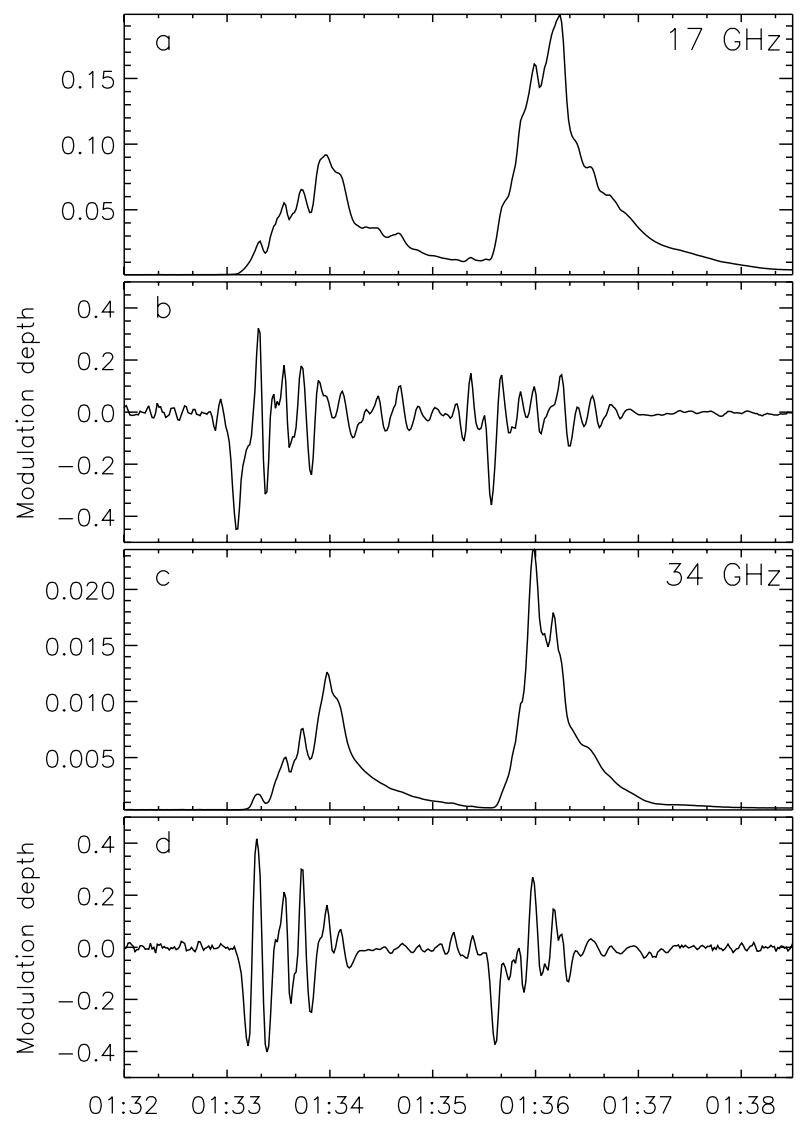

Fig. 3. The averaged correlation amplitude $\rho(t)$ of the signals recorded by $\mathrm{NoRH}$ at $17 \mathrm{GHz}$ a) and $34 \mathrm{GHz}$ c) and relative variations of the signal, $\Delta \rho / \rho=\left(\rho(t)-\rho_{0}\right) / \rho_{0}$, corresponding to the modulation depth at each frequency $\mathbf{b}), \mathbf{d})$.

observed correlation coefficients over the higher spatial frequencies. Consequently, it eliminates background emission and reflects integrated radio fluxes only from fine spatial structures smaller than about 24" (see NoRH Catalog of Events). For both correlation curves, we have analysed the relative change of the signal, $\Delta \rho / \rho=\left(\rho(t)-\rho_{0}\right) / \rho_{0}$, corresponding to the 


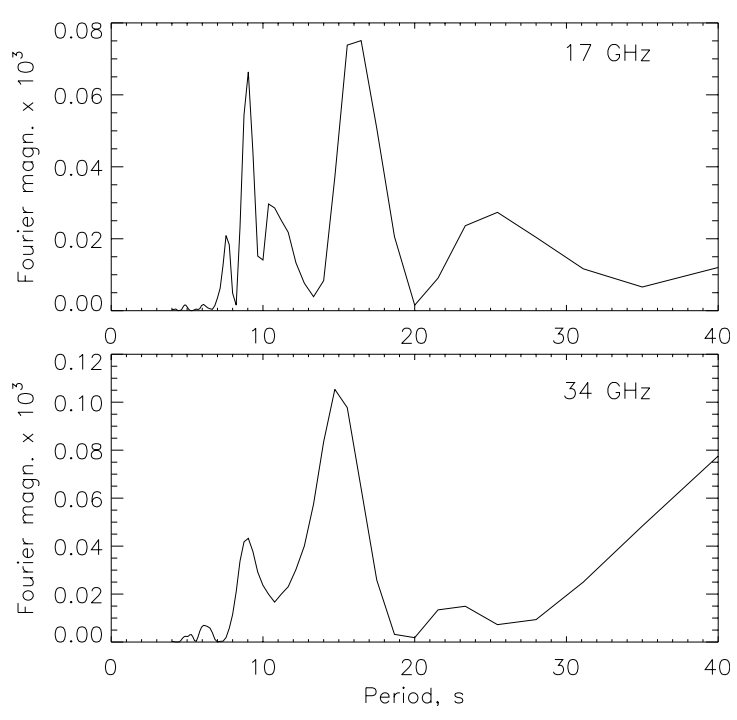

Fig. 4. Fourier spectra of the relative variations $\Delta \rho / \rho=\left(\rho(t)-\rho_{0}\right) / \rho_{0}$ of the NoRH correlation amplitude signal in the interval $\pm 40 \mathrm{~s}$ about 01:36:20 UT at 17 and $34 \mathrm{GHz}$. The spectra show the presence of at least two well pronounced spectral components, with the periods $14-17 \mathrm{~s}$ and $8-10 \mathrm{~s}$.

modulation depth (Figs. 3b and d). The slowly varying mean signal $\rho_{0}$ is obtained by the 10 -s smoothing of the signal. The average modulation depth is about $15 \%$. The Fourier spectral analysis shows that the analysed signal contains wellpronounced periodicities: the strongest of 14-17 s, and another one of about 8-10 s (Fig. 4).

The pulsations are observed to be synchronous at both the frequencies analysed. Moreover, it is worth to note that the microwave pulsations coincide in time with the quasi-periodic pulses detected at the HXR time profiles in the $25-85 \mathrm{keV}$ and $85-826 \mathrm{keV}$ channels of Yohkoh/WBS. Similar to the microwave emission (Fig. 4), the Fourier spectra of the HXR total flux oscillations show the presence of two well pronounced spectral components: with the periods $15-17 \mathrm{~s}$ and $8-9 \mathrm{~s}$ in the $25-85 \mathrm{keV}$ channel and with the periods $13-15 \mathrm{~s}$ and $6-9 \mathrm{~s}$ in the $85-826 \mathrm{keV}$ channel. Roughly, these periods are consistent with the periods found for the integrated microwave emission.

\subsection{Spatial structure of the radio and $X$-ray sources}

The identification of observed modes requires the estimation of physical parameters in the oscillating loop. Figure 5 shows the radio images taken at the flare maximum at 01:36:15 UT at $34 \mathrm{GHz}$ (filled pixels) and $17 \mathrm{GHz}$ (the dotted contour curves), the contour levels are $0.7,0.85$ and 0.98 of the maximum brightness temperature $T_{B 17}$. The brightness temperatures reached values $5.7 \times 10^{7} \mathrm{~K}$ at $17 \mathrm{GHz}$ and $3.7 \times 10^{7} \mathrm{~K}$ at $34 \mathrm{GHz}$. The black contours show the positioning of the elongated HXR source, obtained in the channel L (13.9-22.7 keV) of Yohkoh/HXT, and correspond to the levels 0.2, 0.4, 0.6 and 0.9 of the maximum value. The HXR signal had been integrated over the time interval 01:35:52-01:36:15 UT. The source's double peak structure shows the places of strong electron precipitation.

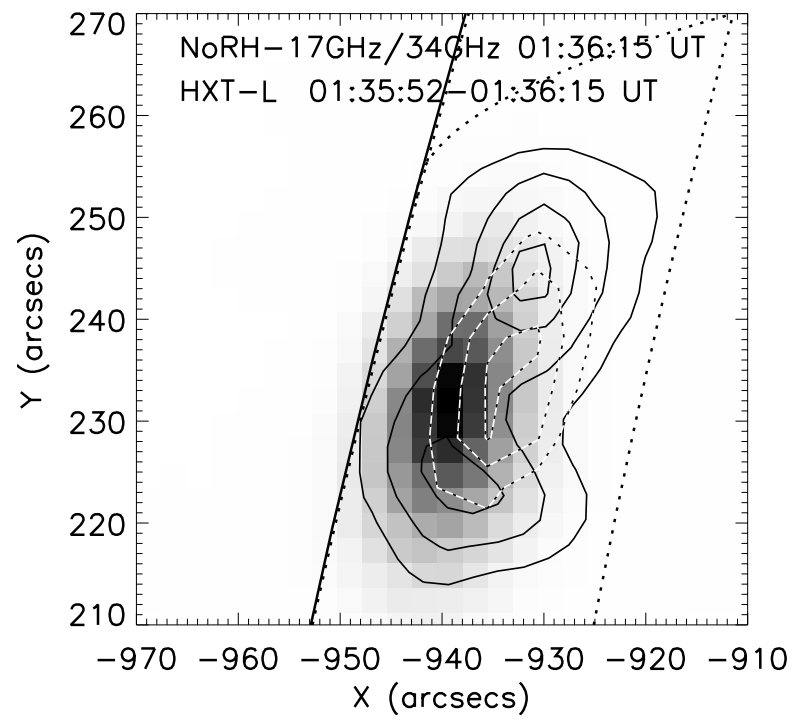

Fig. 5. Radio images made at the flare maximum at 01:36:15 UT at $34 \mathrm{GHz}$ (filled pixels) and $17 \mathrm{GHz}$ (dotted contour curves, the contour levels are $0.7,0.85$ and 0.98 of the maximum brightness temperature $T_{B 17}$ ). The black contour shows the positioning of the HXR sources, obtained in the channel L (13.9-22.7 keV) of Yohkoh/HXT. The HXR signal had been integrated over the time interval 01:35:52-01:36:15 UT, the contours correspond to the levels 0.2 , $0.4,0.6$ and 0.9 of the maximum value.

The radio source projections are seen to be of elliptical shape at both frequencies. We can clearly see the microwave brightness maxima to be located close to the midpoint of the two HXR brightness peaks. This means that the microwave brightness maximum at both frequencies is located near the top of the flaring loop connecting the two HXR brightness maxima. As shown by Melnikov et al. (2002a) such a kind of the microwave brightness distribution is quite common and is explained by the strong concentration of mildly relativistic electrons near the loop top.

An interesting feature of the brightness spatial structure of this specific flare is that the $17 \mathrm{GHz}$ and $34 \mathrm{GHz}$ brightness centres are seen to be displaced with respect to each other. Indeed, the $34 \mathrm{GHz}$ brightness centre is situated definitely (about 2-5") higher than the $17 \mathrm{GHz}$ one (see Fig. 5).

According to the NoRH $34 \mathrm{GHz}$ radio image, the loop length is about $2.5 \times 10^{9} \mathrm{~cm}$ (about $34^{\prime \prime}$ ), and the average width at half intensity is about $6 \times 10^{8} \mathrm{~cm}$ (about $8^{\prime \prime}$ ).

Unfortunately, the observations in the SXR band, with Yohkoh/SXT, were delayed and started 9 min after the peak of the radio burst. The analysis of the images confirms the presence of a magnetic loop that links two HXR brightness peaks corresponding to the loop footpoints.

\subsection{Spatial properties of the pulsations}

As the flaring loop observed was well resolved with NoRH, it made possible to study the properties of the radio pulsations at different parts of the loop. To improve the signal to noise ratio, the signal was integrated over a $10^{\prime \prime} \times 10^{\prime \prime}$ box. Then, for each box, we calculated the flux density $F(t)$, integrating the 

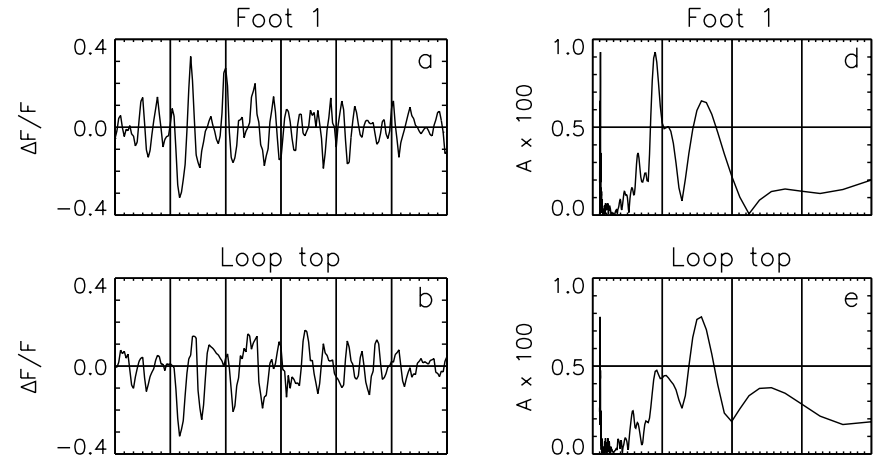

Foot 2
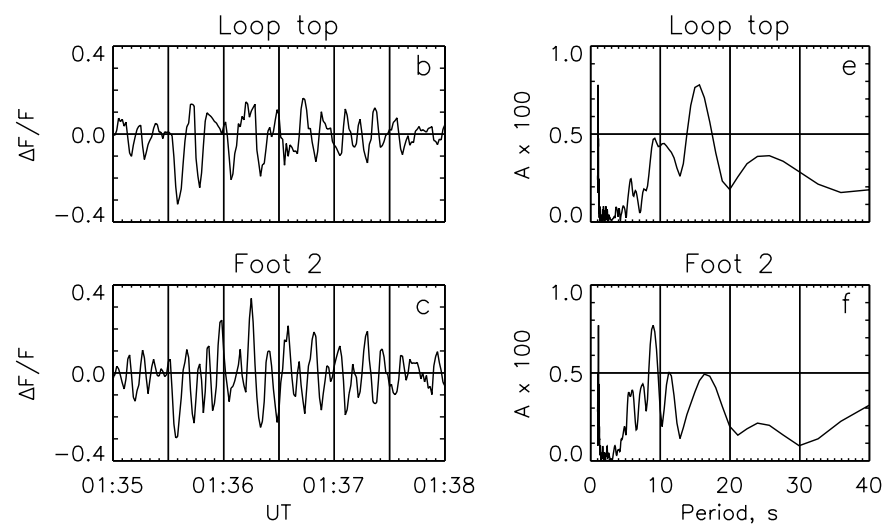

Foot 2

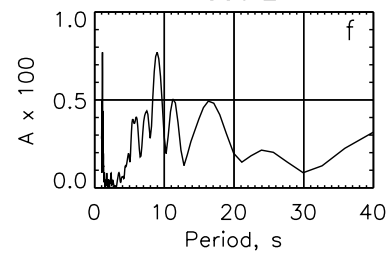

Fig. 6. The time profiles $\Delta F / F$ measured at $17 \mathrm{GHz}$ for the three different regions of the size $10^{\prime \prime} \times 10^{\prime \prime}$ : the Southern (panel a)) and Northern c) legs of the loop, and the loop apex b) determined as the centre of the radio source detected at $34 \mathrm{GHz}$. The Fourier spectra of these time profiles are shown in plots d)-f), respectively. The spectra show that there are both the 8-11 s and 14-17 s spectral components everywhere in the loop, the shorter period component dominates at the legs (d) and f)), while the longer period component - at the apex e).

values of the radio brightness $T_{B}$ over the box in an NoRH image. In addition, the relative variations of the flux $\Delta F / F$ were calculated for each box. Such an analysis revealed that the pulsations were present in the loop wherever we placed the box. Similarly to the integrated flux, the pulsations observed at $17 \mathrm{GHz}$ and $34 \mathrm{GHz}$ are synchronous with each other. And, most interestingly, they are correlated at any part of the loop.

The time profiles $\Delta F / F$ measured at $17 \mathrm{GHz}$ are shown in Fig. 6, for the Southern (panel a) and Northern (c) legs of the loop near the footpoints, and for the loop apex (panel b). The loop apex was determined as the centre of the radio source detected at $34 \mathrm{GHz}$, and the positions of the footpoints - as HXR brightness maxima. The Fourier spectra of those time profiles are shown in Figs. 6d-f, respectively. The pulsations at the loop legs are observed to be well correlated with each other. However, the pulsations observed at the legs do not correlate very well with the pulsations recorded near the apex. Indeed, if some peaks at the leg and apex time profiles coincide with each other, there are certainly more peaks at the leg time profiles than at the apex. The Fourier analysis demonstrates that despite there being both the 8-11 s and 14-17 s spectral components everywhere in the loop, the shorter period component dominates at the legs (Figs. 6d and f), while the longer period component - at the apex (Fig. 6e). The last result coincides with the result of the Fourier analysis of the correlation curves (Figs. 3, 4). This is connected with the fact that the contribution of the apex signal to the correlation flux is strongest, as the apex is seen to be the brightest part of the loop.

A search for a phase shift between oscillations in different parts of coronal loops is very important for selection between
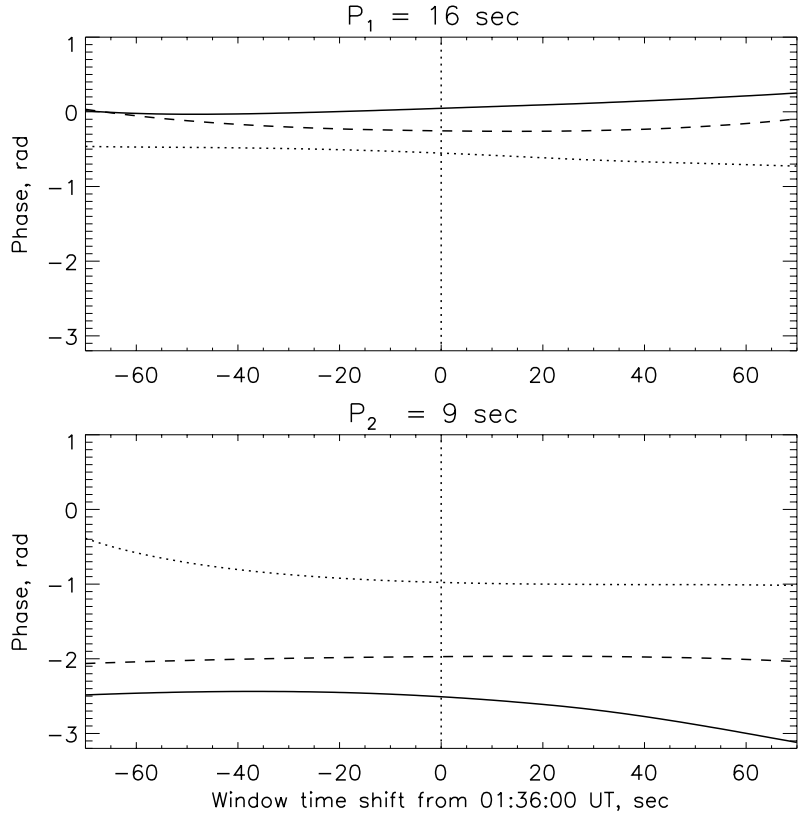

Fig. 7. The phase evolution near the burst maximum at 01:36:00 UT for the two spectral peaks of oscillations: $P_{1}=16 \mathrm{~s}$ and $P_{2}=9 \mathrm{~s}$. The phase of oscillations for the loop top source is shown by the solid line, and for the northern (Foot 2) and southern (Foot 1) leg sources by the dotted and dashed lines, respectively. The phase shift between oscillations from the loop top and foot sources is well pronounced for the $P_{2}$ spectral component and is very small for the $P_{1}$ component.

different MHD modes and their harmonics. In Fig. 7 we compare phases of oscillations for the peak periods of the two spectral components, $P_{1}=16 \mathrm{~s}$ and $P_{2}=9 \mathrm{~s}$. The phases in the interval $\pm 70 \mathrm{~s}$ around the burst maximum 01:36:00 UT were calculated using imaginary and real parts of the Fast Fourier Transform of the oscillating signal in the running window of the Gaussian shape

$g\left(t, t_{0}\right)=\exp \left[-\left(t-t_{0}\right)^{2} / \tau^{2}\right]$

with the width $\tau=90 \mathrm{~s}$ that is comparable with the burst half amplitude duration. The phase of the oscillations for the loop top source is shown by solid line, and for the northern (Foot 2) and southern (Foot 1) leg sources - by dotted and dashed lines, respectively. Phases of both spectral components are quite stable in the time interval $\pm 70 \mathrm{~s}$ around 01:36:00 UT. As one can see, the phase shift between oscillations from the loop top and legs sources is very small for the $P_{1}$ component, less than $0.5 \mathrm{rad}$ or $1 / 12 \times P_{1} \approx 1.3 \mathrm{~s}$, and two times less for the opposite leg sources. However it is well pronounced for the $P_{2}$ spectral component. The value of the phase shift between $P_{2}$ oscillations from the loop top and the northern leg sources is $\approx 1.5 \mathrm{rad}$ or $\approx 1 / 4 \times P_{2}=2.2 \mathrm{~s}$. For the southern leg source the phase difference has the same sign, but the value is three times smaller, only $\approx 0.5 \mathrm{rad}$ or $1 / 12 \times P_{2} \approx 0.7 \mathrm{~s}$. The small phase difference for this source is possibly connected with its proximity to the loop top source (see Fig. 5 and the selection criteria for positioning of the loop top and footpoint boxes in the beginning of Sect. 2.3). 

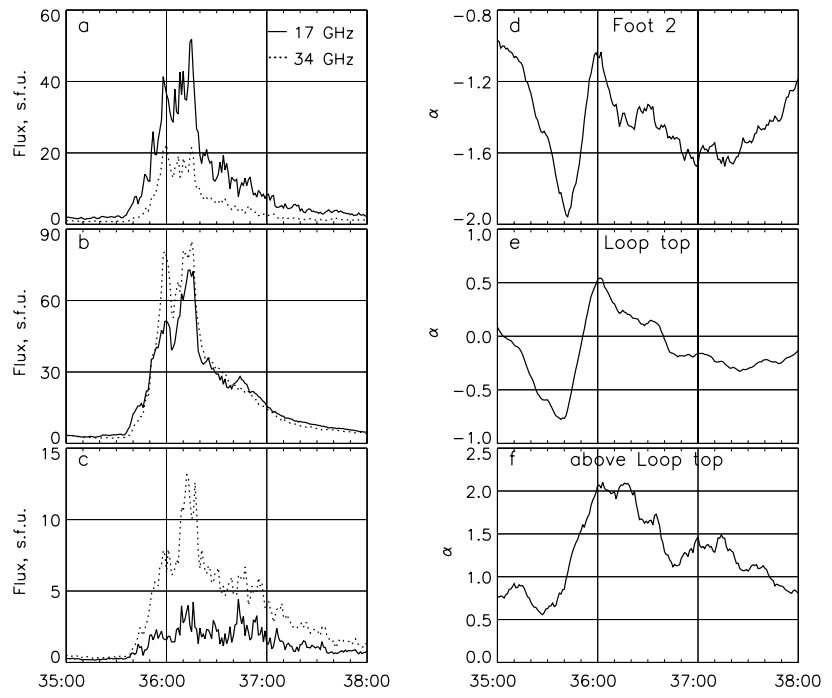

Fig. 8. Left panels: convolved and integrated radio fluxes coming from the $10^{\prime \prime} \times 10^{\prime \prime}$ boxes situated at the centre of the Northern HXR source (the loop footpoint, panel a), at the centre of the $34 \mathrm{GHz}$ source (panel b)) and at the region 20" eastward, i.e. over the loop (panel c)). Right panels: the time profiles of the spectral indices $\alpha$, calculated as $\alpha=\log \left(F_{34} / F_{17}\right) / \log (34 / 17)$, where $F_{34}$ and $F_{17}$ are the fluxes measured at the $34 \mathrm{GHz}$ and $17 \mathrm{GHz}$ bands, which characterise the spectral steepness, are shown in panels $\mathrm{d}$, e and $\mathrm{f}$ (the regions correspond to the time profiles shown in panels a), b) and c)).

\subsection{The spectral index distribution over the source}

Figure 8 shows the integrated radio fluxes coming from the $10^{\prime \prime} \times 10^{\prime \prime}$ boxes situated at the centre of the the Northern HXR source (the loop footpoint, panel a), at the centre of the $34 \mathrm{GHz}$ source, i.e. in the loop top (panel b) and at the region $20^{\prime \prime}$ eastward, i.e. over the loop top (panel c). The time profiles of the parameter $\alpha$, calculated as $\alpha=\log \left(F_{34} / F_{17}\right) / \log (34 / 17)$, where $F_{34}$ and $F_{17}$ are the fluxes measured at the $34 \mathrm{GHz}$ and $17 \mathrm{GHz}$ bands, and which characterise the spectral steepness, are shown in panels $\mathrm{d}$, e and $\mathrm{f}$ (the regions correspond to the time profiles shown in panels $a, b$ and c). One can see that the shapes of the time profiles are similar to each other. However, the values of the spectral parameter $\alpha$ at different parts of the analysed region are very different. Near the loop footpoints, this parameter is negative, $\alpha<0$, while at the upper part of the loop, near the brightness centre and above it is positive, $\alpha>0$. In that part of the analysed region, the $34 \mathrm{GHz}$ flux is significantly stronger than the $17 \mathrm{GHz}$ flux. The spectral in$\operatorname{dex} \alpha$ remains high at the region above the brightness centre at $34 \mathrm{GHz}$, where the brightness temperature diminishes down to $T_{B}=10^{5}-10^{6} \mathrm{~K}$. Along with this, we should mention that $\alpha$ becomes negative below the brightness centre of the loop similarly to the regions near footpoints.

\section{Diagnostics of physical conditions in the loop}

It is commonly accepted that the intensive $\mathrm{mm}$ - and $\mathrm{cm}$ emission is generated in a flaring loop by the gyrosynchrotron emission of nonthermal electrons. The spectrum of such emission usually has a characteristic shape, with a single peak at the peak frequency $f_{\text {peak }}$ (Fig. 2). The high frequency turnover of this peak is due to the emission becoming optically thin, with the spectral slope being determined chiefly by the electron energy distribution, while the low frequency slope may be determined by self-absorption (Twiss 1954), cyclotron absorption, or Razin suppression (Razin 1960a,b).

In the case of self-absorption, the frequency of the spectral maximum, $f_{\text {peak }}$, together with the peak flux, $F_{\text {peak }}$, gives the information about the magnetic field strength and column number density of nonthermal electrons. In the case of strong Razin suppression these parameters can also provide information about plasma density in the loop (Fleishman \& Melnikov 2003).

Observations without spatial resolution (see Figs. 1 and 2) indicate that the flux density spectral peak is located between 17 and $34 \mathrm{GHz}$. From observations with spatial resolution, showing almost equal brightness temperatures at both frequencies near the loop top, we conclude that the flux density has the maximum at $f_{\text {peak }}>34 \mathrm{GHz}$ or in the interval between 17 and $34 \mathrm{GHz}$. The first is true if the source is resolved across the loop at both frequencies (its size is greater than the beam size, $10^{\prime \prime}$ ), so that the brightness temperatures measured at 17 and $34 \mathrm{GHz}$ are real. The second is true if the actual width of the source at $17 \mathrm{GHz}$ is less than the beam size. Near the footpoints, the spectral peak should be located at the frequency $f \leq 17 \mathrm{GHz}$ (since the spectral slope between 17 and $34 \mathrm{GHz}$ is negative). Consequently, it is quite likely that the source is optically thin in this region.

The origin of the low frequency turnover at the loop top is very important for understanding the physical conditions in the loop considered. According to SOHO/MDI images, the photospheric magnetic field did not exceed $B=100 \mathrm{G}$ around the south footpoint and $B=400 \mathrm{G}$ around the north footpoint on the 12th of January, as well as on the 14th of January, when the observed region had significantly moved from the limb and the MDI measurement precision became sufficient. It means that the spectral peak was at least at the 20th harmonic of gyrofrequency. This is certainly not likely for the effective cyclotron absorption, and therefore, one can neglect this mechanism for the low frequency turnover.

The role of the gyrosynchrotron self-absorption is also problematic for this event. If the spectral depression at $17 \mathrm{GHz}$ was connected with enhanced optical thickness, $\tau \geq 1$, at $f \leq 17 \mathrm{GHz}$, the modulation depth of the emission would be much less pronounced at low frequencies. Besides, the time profiles of the emission intensity would be smoother than they are at $34 \mathrm{GHz}$. Moreover, in the case when the modulation of the flux is due to temporal magnetic field variations in the microwave source, the oscillations of the flux at low frequencies $f<f_{\text {peak }}$ would be in anti-phase with the oscillations at high frequencies, $f>f_{\text {peak }}$, since for the gyrosynchrotron emission $F\left(f<f_{\text {peak }}\right) \propto B^{-\beta_{1}}, \beta_{1}=0.5-1$, but $F\left(f>f_{\text {peak }}\right) \propto B^{\beta_{h}}$, $\beta_{h}=3-5$. However, the observations show just opposite: the modulation depth at $f \leq 17 \mathrm{GHz}$ is even higher than they are at $34 \mathrm{GHz}$, and the oscillations are in phase at low, $f<f_{\text {peak }}$, and high, $f>f_{\text {peak }}$ frequencies.

Another important difficulty for the explanation of the lowfrequency turnover by the self-absorption is the combination of the high value of $f_{\text {peak }}$ and the relatively low brightness 


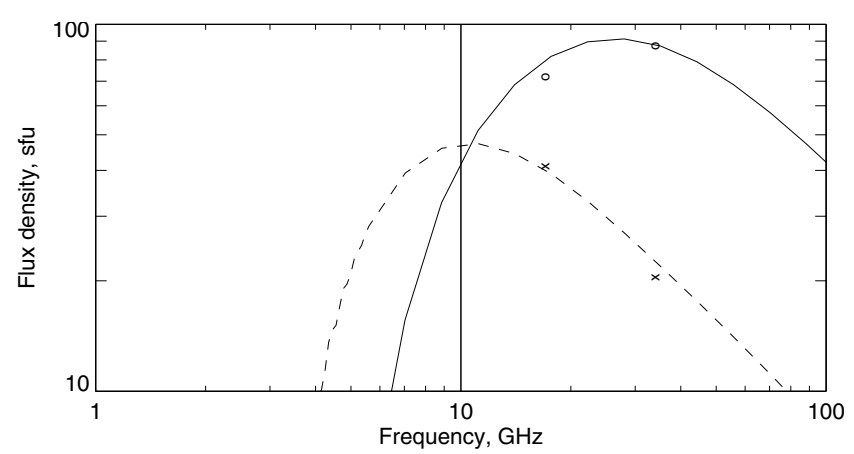

Fig. 9. Comparison of observed fluxes at 17 and $34 \mathrm{GHz}$ from the loop top (circles) and footpoint (crosses) source with $10^{\prime \prime} \times 10^{\prime \prime}$ area and the corresponding microwave spectra calculated for the sources of the same size. The plasma density is assumed constant along the loop, $n_{0}=8 \times 10^{10} \mathrm{~cm}^{-3}$, the magnetic field is $100 \mathrm{G}$ and $200 \mathrm{G}$ in the loop top and footpoint sources, respectively. The electron power law spectral index is $\delta=3.0$, the number density of energetic electrons in the loop top is $n_{\mathrm{lt}}(E>1 \mathrm{MeV})=6.1 \times 10^{4} \mathrm{~cm}^{-3}$ and near the footpoint $n_{\mathrm{fp}}(E>1 \mathrm{MeV})=2.3 \times 10^{3} \mathrm{~cm}^{-3}$.

temperature at both 17 and $34 \mathrm{GHz}$. The observed value of the brightness temperature, $T_{\mathrm{b}} \sim(3-6) \times 10^{7} \mathrm{~K}$, is at least 10 times lower than expected from gyrosynchrotron theory even if one assumes that 1) the magnetic field in the loop top is equal to the maximum magnetic field in the foot points, $B=400 \mathrm{G}$, and 2) the electron spectral index is very high, e.g. $\delta=5.3$ as follows from Yohkoh HXT and WBS data on the hard X-ray spectral slope at low energies $(<100 \mathrm{keV})$ in the thick target approach $\left(\gamma_{x}=4.3\right)$. For a more realistic field strength near the loop top $B \approx 100 \mathrm{G}$ and lower $\delta$ (e.g. $\delta \approx 3$ as follows from the microwave spectral slope), the discrepancy reaches two orders of magnitude. Our model simulations show that the spectral peak observed between 17 and $34 \mathrm{GHz}$ in the top part of the loop cannot be explained by the self-absorption with any reasonable assumptions on the value of the magnetic field strength and nonthermal electron energy spectral slope. The only theoretical possibility is the assumption that the source size at 34 and $17 \mathrm{GHz}$ was much less (about 10 times) than the beam size. But this assumption definitely does not work since the the NoRH observations show that the source is well resolved at least in the longitudinal direction.

An alternative mechanism for the low frequency turnover is Razin suppression which is efficient in the situation when there is a rather large ratio of plasma density to magnetic field. This effect has been shown to be quite common in solar flaring loops (Melnikov et al. 2005). Assuming that the Razin effect indeed takes place, we obtain a good agreement between theory and observed spectra and brightness temperatures in different parts of the flaring loop (the loop top and parts of the legs near footpoints). This agreement is valid for the plasma density close to $n_{0}=10^{11} \mathrm{~cm}^{-3}$ and magnetic field in the range $B \sim 50-100 \mathrm{G}$ (depending on an electron spectral index at relativistic energies). The low value of the magnetic field at the loop top is in good agreement with low photospheric magnetic field in the corresponding active region $(\leq 100 \mathrm{G}$ around the south footpoint and $\leq 400 \mathrm{G}$ around the north footpoint).
In Fig. 9 we show the gyrosynchrotron spectra calculated for the loop top and footpoint conditions. Here, we used the full gyrosynchrotron formalism (Ramaty 1969; Fleishman \& Melnikov 2003). For the estimation purpose, we assume that the electron distribution is isotropic. This assumption is justified for the considered limb flare loop which has the angle between the magnetic field and the line-of-sight close to $90^{\circ}$ at all its parts. In addition, we make a reasonable assumption that the magnetic field strength increases from $100 \mathrm{G}$ at the loop top to $200 \mathrm{G}$ at the footpoint, the plasma density is constant along the loop, the angle between the magnetic field and the line-of-sight is 78.5 degree, the source depth is $d=6 \times 10^{8} \mathrm{~cm}$, and the electron energy spectrum is given by a single power law in the range $E=100-31600 \mathrm{keV}$. The best fits to the observed fluxes from the $10^{\prime \prime} \times 10^{\prime \prime}$ areas at 17 and $34 \mathrm{GHz}$ have been obtained for the following conditions: the plasma density $n_{0}=8 \times 10^{10} \mathrm{~cm}^{-3}$, the electron spectral index $\delta=3.0$, the number density of energetic electrons in the loop top and footpoint parts $n_{\mathrm{lt}}(E>1 \mathrm{MeV})=6.1 \times 10^{4} \mathrm{~cm}^{-3}$ and $n_{\mathrm{fp}}(E>1 \mathrm{MeV})=2.3 \times 10^{3} \mathrm{~cm}^{-3}$, respectively. In Fig. 9, the solid line shows the frequency spectrum of the microwave flux density from the box $10^{\prime \prime} \times 10^{\prime \prime}$ located in the upper part of the simulated magnetic loop. The dashed line shows the spectrum from a similar area located in the lower part of the loop. We can clearly see that for the lower part of the loop the spectral peak shifts to lower frequencies and the spectral slope between 17 and $34 \mathrm{GHz}$ changes from positive to negative. The physical reason for this shift is the increase up to the factor of two in the magnetic field strength near the footpoint and, therefore, the significant decrease of Razin suppression at $17 \mathrm{GHz}$. This theoretical prediction explains very well the observed spatial distribution of the spectral slope (parameter $\alpha$ in Figs. 8a-d, b-e). Indeed, near the north footpoint, where the magnetic field is expected to be at least 2 times higher than at the loop top, the observed spectral slope is negative, i.e. $I(17 \mathrm{GHz}) \gg I(34 \mathrm{GHz})$, during the whole burst.

Another consequence of the decrease of the ratio $B / n_{0}$ with height (increase of Razin suppression) is the spatial shift of the brightness peaks between 17 and $34 \mathrm{GHz}$. To illustrate this shift, we calculated the spatial profiles of the intensity across the loop top for a model flaring loop (see Fig. 10). The zero position is associated with the upper edge of the chromosphere. The displacement of the 17 and $34 \mathrm{GHz}$ intensity maxima is clearly seen in Fig. 10b. It explains well the observed displacement seen in Fig. 6. For the simulations we assumed the Gaussian distribution of plasma density and high energy electrons (dot-dashed and dotted lines in Fig. 10a). The maximum values of plasma and energetic electron number densities are taken to be the same as above for the loop top source (Fig. 9). The magnetic field strength is $100 \mathrm{G}$ at the loop axes and slowly changes with height as $B=120\left(s_{1} /\left(s+s_{1}\right)\right)^{3}$, where $s_{1}=9.6 \times 10^{9} \mathrm{~cm}$. It is interesting that the spectral slope $\alpha$ once again changes from negative values below the loop axes to positive ones at the axes and above it in agreement with observations (see Figs. 8c and d).

Obviously, the high value of the spectral parameter $\alpha$ above the loop and the observed shift of the brightness peaks at 17 and $34 \mathrm{GHz}$ cannot be explained by effects of the gyrosynchrotron 

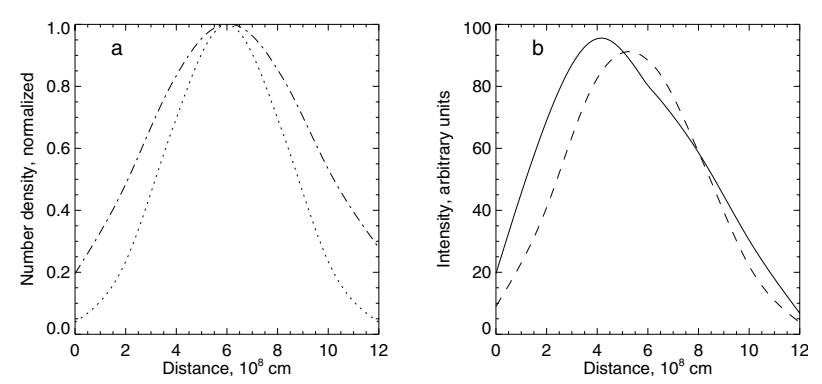

Fig. 10. a) Distribution of plasma density (dot-dashed) and high energy electrons (dotted line). The maximum value of number densities of plasma and energetic electrons are taken the same as for the loop top source (Fig. 9). b) Intensity spatial profiles across the top part of a model flaring loop. The displacement of 17 (solid line) and $34 \mathrm{GHz}$ (dashed line) intensity maxima due to Razin suppression of $17 \mathrm{GHz}$ emission near the loop axes is clearly seen.

self-absorption because the brightness temperature at $17 \mathrm{GHz}$ drops down here by an order of magnitude with respect to the loop top.

The high values of plasma density in the loop $\left(n_{0} \approx\right.$ $10^{11} \mathrm{~cm}^{-3}$ ) obtained above are also confirmed by the short decay times $(<2-5 \mathrm{~s})$ for major burst subpeaks and undetectable $(<1 \mathrm{~s})$ time delays between microwave and hard X-ray peaks and between the peaks at 17 and $34 \mathrm{GHz}$ (which are usually observed in larger flaring loops due to the difference between the energy-dependent collisional life times of electrons responsible for the hard X-ray and 17 and $34 \mathrm{GHz}$ emission, see Melnikov et al. 2002c). Estimations of the decay times $\tau_{\text {dec }}$ of the relativistic electron number density $N(E, t) \propto E^{-\delta}$, responsible for the microwave emission at the observed frequencies show that these decay times are in agreement with the value of plasma density $n_{0}=10^{11} \mathrm{~cm}^{-3}$.

In addition, the high value of plasma density $n_{0}$ inside the flaring loop is confirmed by the analysis of integral soft $\mathrm{X}$ ray burst fluxes recorded by GOES in two channels $1-8 \AA$ and $0.5-4 \AA$. In this analysis we have used the standard SSW procedures and got the values of the temperature, $T=10-15 \times 10^{6} \mathrm{~K}$, and the emission measure

$E M=\int n_{0}^{2} \mathrm{~d} V \approx 25 \times 10^{48} \mathrm{~cm}^{-3}$

at the time of the microwave burst maximum 01:36:00 UT. Taking into account that the flaring loop sizes at that moment were $L=25 \times 10^{8} \mathrm{~cm}$ and $a=3 \times 10^{8} \mathrm{~cm}$, respectively, and calculating the volume of the loop as $V=\pi a^{2} L$, we obtain the estimate of the plasma density, $n_{0}=(E M / V)^{1 / 2} \approx 1.8 \times 10^{11} \mathrm{~cm}^{-3}$. This value is almost two times higher than the estimates obtained with other methods, but still in agreement with them within the limits of the method's inaccuracy.

The high density of the loop plasma is also in agreement with the density estimations obtained with the use of Yohkoh/SXT images of the postflare loop taken nine minutes after the burst maximum. We calculated the column emission measure per pixel at the location of $34 \mathrm{GHz}$ brightness peak (loop top)

$E M=S_{\text {pix }} \int n_{0}^{2} \mathrm{~d} l \approx S_{\text {pix }} n_{0}^{2} l$ where the pixel area is $S_{\text {pix }}=2.5^{\prime \prime} \times 2.5^{\prime \prime}$, and the source depth along the line-of-sight, $l$, is taken to be equal to the measured width of the loop, $6 \times 10^{8} \mathrm{~cm}$. For the calculations we used the standard routine SXT_TEEM. The column EM in the postflare loop reaches the value $1.7 \times 10^{47} \mathrm{~cm}^{-3}$ at the time near 01:50 UT. It corresponds to $n_{0} \approx 10^{11} \mathrm{~cm}^{-3}$.

\section{Mechanisms for the microwave pulsations}

The most probable mechanisms for the quasi-periodic modulation of the emission intensity could be a) variations of the magnetic field strength in the loop; b) variations of the angle between the line-of-sight and the magnetic field vector; c) variations of the mirror ratio in the loop, modulating the loss cone and, therefore, the electron precipitation; and d) a quasiperiodic regime of the energetic electron acceleration/injection in the loop. In any case, the period of the observed oscillations (of order of $10 \mathrm{~s}$ ) strongly indicates the MHD nature of the pulsations. Indeed, MHD modes of the flaring loop can modulate the efficiency of the gyrosynchrotron emission of the electrons in the loop or affect the electron acceleration, injection or accumulation/precipitation in the loop.

Assuming that the pulsations are produced by variation of the value and direction of the magnetic field, and taking into account the observed modulation depth of the radio pulsations, 15-20\%, we can estimate the amplitude of the magnetic field perturbations. For the estimations we use Dulk \& Marsh's (1982) approximated formula for the intensity, $I_{f}$, of the optically thin gyrosynchrotron emission at a frequency $f$.

Under the assumption that the direction of the magnetic field in the loop does not change (e.g., sausage modes to be discussed in Sect. 5) and that the pulsations are connected with periodic expansions and contractions of the loop tube, we express the relationship of the intensity variation with the magnetic field strength $B$,

$I_{f} \propto B^{0.90 \delta-0.22}$

which for the electron spectral index $\delta=5$ gives the relative perturbation of the magnetic field $\Delta B / B \approx 3-4 \%$.

On the other hand, if we assume that the pulsations are associated with the variations of the angle $\theta$ between the magnetic field and the line-of-sight and that the absolute value of the field does not change (e.g., kink modes, see Sect. 5), the expression for the intensity reduces to

$I_{f} \propto(\sin \theta)^{-0.43+0.65 \delta}$

Taking $\theta \approx 80^{\circ}$, as the flare takes place at the limb, we obtain that $\Delta \theta \approx 12^{\circ}-15^{\circ}$ or, for the relative variation of the angle, $\Delta \theta / \theta \approx 15 \%-19 \%$. This value seems to be rather too high, especially if we take into account that these variations can affect the emission only for the source located near footpoints. For the loop top source the viewing angle practically does not change, as the loop is on the limb.

The estimations above are suitable only for optically thin regions. For the source considered this condition is fulfilled for sure for the regions near the loop footpoints and below the source centre, where the spectral index $\alpha$ is observed to be negative. At and above the centre, $\alpha>0$ and, consequently, those 
regions may be optically thin only in the case of strong Razin suppression. If the loop top source is optically thick, the intensity variations caused by the fluctuations of the magnetic field strength,

$I_{f} \propto B^{-0.50-0.085 \delta}$,

would occur in anti-phase with variations at higher frequencies and they must be several times weaker, which contradicts with our observations. Consequently, the applicability of the optically thin model is justified.

\section{Modes of MHD-oscillations of coronal loops}

According to the theory developed by Edwin \& Roberts (1982, 1983) (see also Zaitsev \& Stepanov 1975) linear MHD perturbations of a straight plasma cylinder with the radius $r=a$ are described by dispersion relation

$\rho_{\mathrm{e}}\left(\omega^{2}-k_{z}^{2} C_{\mathrm{Ae}}^{2}\right) m_{0} \frac{I_{m}^{\prime}\left(m_{0} a\right)}{I_{m}\left(m_{0} a\right)}$

$+\rho_{0}\left(k_{z}^{2} C_{\mathrm{A} 0}^{2}-\omega^{2}\right) m_{\mathrm{e}} \frac{K_{m}^{\prime}\left(m_{\mathrm{e}} a\right)}{K_{m}\left(m_{\mathrm{e}} a\right)}=0$,

where $I_{m}(x)$ and $K_{m}(x)$ are modified Bessel functions of order $m$, and the prime denotes the derivative with respect to argument $x$. The functions $m_{0}$ and $m_{\mathrm{e}}$ which may be considered as radial wave numbers of the perturbations inside and outside the cylinder, respectively, are defined through

$m_{i}^{2}=\frac{\left(k_{z}^{2} C_{\mathrm{si}}^{2}-\omega^{2}\right)\left(k_{z}^{2} C_{\mathrm{A} i}^{2}-\omega^{2}\right)}{\left(C_{\mathrm{s} i}^{2}+C_{\mathrm{A} i}^{2}\right)\left(k_{z}^{2} C_{\mathrm{T} i}^{2}-\omega^{2}\right)}$,

where $i=0, e$. In the internal and external media, the sound speeds are $C_{\mathrm{s} 0}$ and $C_{\mathrm{se}}$, the Alfvén speeds are $C_{\mathrm{A} 0}$ and $C_{\mathrm{Ae}}$, and the tube speeds are $C_{\mathrm{T} 0}$ and $C_{\mathrm{Te}}$, respectively. (The definitions of the speeds are standard, see, e.g., Nakariakov 2003.) Relations between those characteristic speeds determine properties of MHD modes guided by the cylinder. For modes that are confined to the tube (evanescent outside, in $r>a$ ), the condition $m_{\mathrm{e}}>0$ has to be fulfilled. The integer $m$ is the azimuthal mode number. It determines the azimuthal modal structure: waves with $m=0$ are sausage modes, waves with $m=1$ are kink modes, waves with higher $m$ are referred to as flute or ballooning modes. The existence and properties of the modes are determined by the equilibrium physical quantities. In particular, for $m_{0}^{2}<0$, the internal radial structure of the modes is described by the Bessel functions $J_{m}(x)$, and the radial dependence of the oscillation is quasi-periodic.

Figure 11 shows dispersive curves of MHD modes guided by a magnetic cylinder with the characteristic speeds $C_{\mathrm{A} 0}=$ $600 \mathrm{~km} \mathrm{~s}^{-1}, C_{\mathrm{Ae}}=3300 \mathrm{~km} \mathrm{~s}^{-1}, C_{\mathrm{s} 0}=340 \mathrm{~km} \mathrm{~s}^{-1}$ and $C_{\mathrm{se}}=$ $200 \mathrm{~km} \mathrm{~s}^{-1}$ that are obtained using the loop parameters derived from microwave and X-ray diagnostics in Sect. 3. There is an infinite number of modes with a given azimuthal number $m$, corresponding to different radial numbers, $l$. The modes with higher radial numbers $l$ have higher phase speeds along the loop and exist for higher values of the longitudinal wave number $k$.

The horizontal axis of Fig. 11 shows the normalised longitudinal wave number, which may be rewritten through the longitudinal wave length $k a=2 \pi a / \lambda$. As fast modes of a coronal

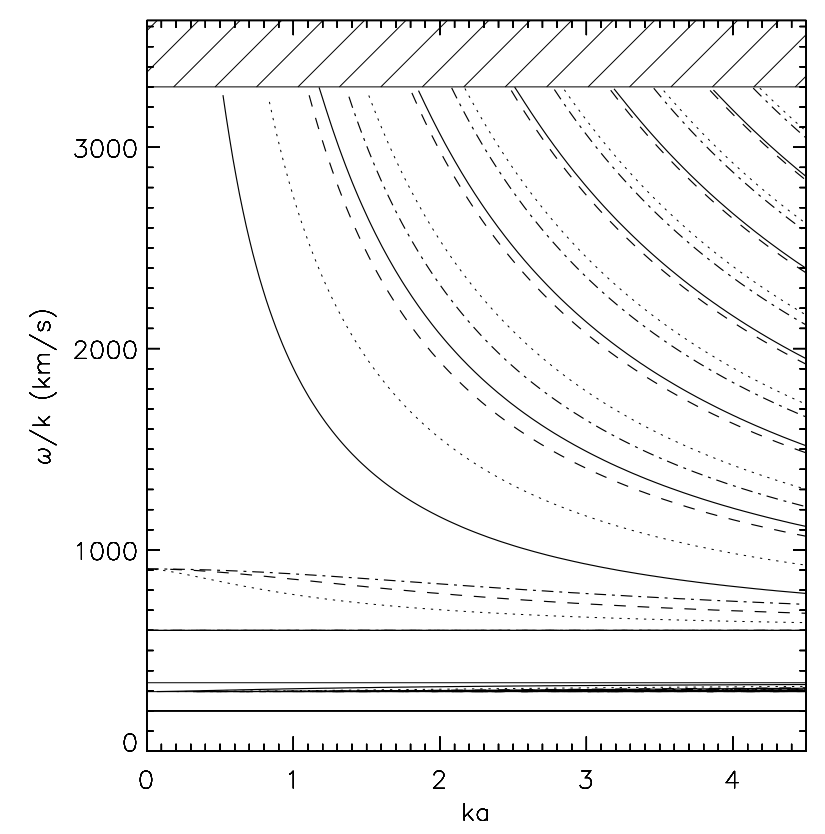

Fig. 11. Phase speeds of MHD modes of a straight magnetic cylinder as function of the longitudinal wave number normalised by the cylinder radius $a$, for $C_{\mathrm{A} 0}=600 \mathrm{~km} \mathrm{~s}^{-1}, C_{\mathrm{Ae}}=3300 \mathrm{~km} \mathrm{~s}^{-1}, C_{\mathrm{s} 0}=$ $340 \mathrm{~km} \mathrm{~s}^{-1}$ and $C_{\mathrm{se}}=200 \mathrm{~km} \mathrm{~s}^{-1}$. Solid curves correspond to sausage $(m=0)$ modes of different radial numbers $(l=1,2,3, \ldots)$, dotted curves to kink $(m=1)$ modes, dashed and dashed-dotted curves - to the ballooning modes $m=2$ and $m=3$, respectively. For fast modes, the lowest phase speed curves of each line style correspond to the $l=1$ mode. Horizontal straight lines show the characteristic speeds mentioned above. The hatched region shows where no trapped mode can exist.

loop should have nodes of the transverse velocity perturbations at the loop footpoints, the wave lengths of the modes are quantized, $\lambda=2 L / n$, where $n$ is an integer corresponding to the mode number (or the number of maxima of the transverse velocity perturbations along the loop) and $L$ is the length of the loop. Identification of a particular mode should be based upon the analysis of the mode period and the mode radial and longitudinal numbers. The radial mode number $l$ is perhaps the most difficult to measure, as the loop width is usually either unresolved or resolved very poorly. The lowest $n=1$ mode is a fundamental or global mode. Global kink $(n=1, m=1)$ modes have been discovered with TRACE EUV imaging telescope (Aschwanden et al. 1999; Nakariakov et al. 1999).

The length of the analysed loop is estimated as $L=25 \mathrm{Mm}$. The observationally determined distribution of the oscillation power along the loop has one maximum, near the loop apex, suggesting that the observed mode is global. In the loop of the observed length, the global $(n=1)$ mode with the period of $16 \mathrm{~s}$ should have the phase speed about $3130 \mathrm{~km} \mathrm{~s}^{-1}$. According to Fig. 11 the longest wave length mode which can have such a speed is the sausage mode. The corresponding normalised longitudinal wave number $k a \approx 0.54$, which gives us $a \approx L / 6 \approx 4.3 \mathrm{Mm}$. As the observed loop is sufficiently thick, this estimation is realistic. The next longest wave length global mode, the kink $l=2$ mode, requires $k a=0.85$, which does not seem to be realistic as the loop would be too thick in this 
Table 1. Phase speeds $V_{p}$ and periods $P$ of MHD modes of a coronal loop with $C_{\mathrm{A} 0}=600 \mathrm{~km} \mathrm{~s}^{-1}, C_{\mathrm{Ae}}=3300 \mathrm{~km} \mathrm{~s}^{-1}, C_{\mathrm{s} 0}=340 \mathrm{~km} \mathrm{~s}^{-1}$ and $C_{\mathrm{se}}=200 \mathrm{~km} \mathrm{~s}^{-1}$. The loop radius is $4.3 \mathrm{Mm}$ and the length is $25 \mathrm{Mm}$. Modes with lowest azimuthal $m$ and radial $l$ numbers and with longest longitudinal wave lengths $\lambda$ are considered only.

\begin{tabular}{ccccccr}
\hline \hline$n$ & $m$ & $l$ & $k a$ & $\lambda / \mathrm{Mm} V_{p} /\left[\mathrm{km} \mathrm{s}^{-1}\right]$ & $P / \mathrm{s}$ \\
\hline 1 & 0 & 1 & 0.54 & 50 & 3128 & 16.0 \\
1 & 1 & 1 & 0.54 & 50 & 836 & 59.8 \\
1 & 2 & 1 & 0.54 & 50 & 886 & 56.4 \\
1 & 3 & 1 & 0.54 & 50 & 897 & 55.7 \\
\hline 2 & 0 & 1 & 1.09 & 25 & 1775 & 14.1 \\
2 & 1 & 1 & 1.09 & 25 & 768 & 32.5 \\
2 & 1 & 2 & 1.09 & 25 & 2549 & 9.8 \\
2 & 2 & 1 & 1.09 & 25 & 847 & 29.5 \\
2 & 2 & 2 & 1.09 & 25 & 3299 & 7.6 \\
2 & 3 & 1 & 1.09 & 25 & 876 & 28.5 \\
\hline 3 & 0 & 1 & 1.64 & 16.7 & 1323 & 12.6 \\
3 & 0 & 2 & 1.64 & 16.7 & 2460 & 6.8 \\
3 & 1 & 1 & 1.64 & 16.7 & 723 & 23 \\
3 & 1 & 2 & 1.64 & 16.7 & 1816 & 9.2 \\
3 & 1 & 3 & 1.64 & 16.7 & 3033 & 5.5 \\
3 & 2 & 1 & 1.64 & 16.7 & 807 & 20.6 \\
3 & 2 & 2 & 1.64 & 16.7 & 2292 & 7.3 \\
3 & 3 & 1 & 1.64 & 16.7 & 850 & 19.6 \\
3 & 3 & 2 & 1.64 & 16.7 & 2771 & 6.0 \\
\hline
\end{tabular}

case. All other global modes require the loop to be even thicker and should be ruled out on those grounds. Similarly, the longitudinal modes are excluded because they cannot reach the required phase speed. In addition, according to Fig. 7 (upper panel) all parts of the loop analysed are observed to oscillate in phase with each other. This confirms the identification of the observed mode as the global sausage $l=1$ mode, as suggested in Nakariakov et al. (2003).

It is more difficult to interpret the shorter period mode observed with the period of $8-11 \mathrm{~s}$. To have such a period, the mode should have much shorter longitudinal wave length than the global mode. Because of the very steep dependence of the phase speed of the sausage mode upon the longitudinal wave number, it is not likely that the observed $9 \mathrm{~s}$ periodicity is associated with the second longitudinal harmonics of this mode. Table 1 shows phase speeds and periods of the modes which exist for the longitudinal wave numbers $k a \approx 1$ and $k a \approx 1.5$, corresponding to the second $(n=2)$ and third $(n=3)$ harmonics of the global mode determined above. As one can see, the second and the third longitudinal harmonics of the kink mode $m=1, l=2$ have periods in the required range $(9.8 \mathrm{~s}$ and $9.2 \mathrm{~s}$, respectively). However, other modes, e.g. the third harmonics of the sausage mode (with the period $12.6 \mathrm{~s}$ ) and the second harmonics of $m=2, l=2$-ballooning mode (7.6 s) should not be ruled out, because the dispersion relation is very sensitive to the uncertainties in characteristic speeds. As the phase shift observed along the loop (see Fig. 7, lower panel) is by a factor of 2 smaller than the expected phase shift between maxima in a mode with $n>1$, we interpret it in favour of a mode with a more complicated radial structure $(l>1)$. In this case, there can be maxima and minima in the radial direction at almost the same position along the loop and the observed phase shift can be smaller than half of the period because of the integration effect. The suitable modes are, e.g., $(n=2, m=1, l=2),(n=2$, $m=2, l=2)$ and $(n=3, m=1, l=2)$.

The theory discussed above does not take into account another MHD mode, the torsional one. Torsional waves are twistings of the tube, propagating at the local Alfvén speed. They can easily be observed via modulation of the gyrosynchrotron emission produced by the local variations of the angle between the magnetic field and the LOS. In a coronal loop, the resonant periods of the torsional mode are given by the expression

$P=2 L / n C_{\mathrm{A} 0}$.

A first possible identification of a global torsional mode has been discussed by Zaqarashvili (2003). In the considered loop, the resonant periods of lower torsional harmonics are $83 \mathrm{~s}$, $42 \mathrm{~s}$ and $27.8 \mathrm{~s}$ for $n=1,2$ and 3, respectively. To give the observed periodicities, the suitable mode numbers are $n=5$ $(P=16.8 \mathrm{~s})$ and $n=6(P=13.9 \mathrm{~s})$. The oscillations with the period in the range $8-11 \mathrm{~s}$ could be associated with $n=8-11$ $(P=10.4-7.6 \mathrm{~s})$. However, it is difficult to explain why a mode with a particular high number is excited only. Also, the wavelength of higher- $n$ modes is much smaller than the pixel size of NoRH, and the positive and negative semi-periods would cancel each other. Consequently, it is not likely that torsional modes are responsible for the observed periodicities.

\section{Conclusions}

In this paper we present one of the first spatially resolved observations of microwave pulsations in a flaring loop (see also Asai et al. 2001; Melnikov et al. 2002b; Grechnev et al. 2003). Observations without spatial resolution could not allow for the confident identification of specific oscillation modes because of too many free parameters. The identification of the oscillation mode should be based upon the determination of the observational period, the longitudinal and transverse sizes of the magnetic loop, and the spatial distribution of the oscillation amplitude and phase along the loop, as well as the plasma density and magnetic field strength inside and outside the loop.

The observations of a flaring loop occurred close to the East solar limb on 12 January 2000 revealed the following properties.

1) Time profiles of the microwave emission at 17 and $34 \mathrm{GHz}$ exhibit quasi-periodical (with two well defined periods $P_{1}=14-17 \mathrm{~s}$ and $P_{2}=8-11 \mathrm{~s}$ ) variations of the intensity in different parts of the loop. The first Fourier spectral peak, $P_{1}$, is more pronounced at the apex while the second one, $P_{2}$, is relatively stronger at the loop legs (near the footpoints). The pulsations at both loop legs are well correlated with the quasi-period $P_{2}=8-11 \mathrm{~s}$, while the correlation between the pulsations at the legs and at the apex is not so pronounced. The 14-17 s pulsations are synchronous at the top and in both legs of the loop. For the $P_{2}$ spectral component, a definite phase shift, $P_{2} / 4 \approx 2.2 \mathrm{~s}$, between pulsations in the northern leg and top part of the loop were found. 
2) The length of the flaring loop is estimated as $L=25 \mathrm{Mm}$ and its average width at half intensity at $34 \mathrm{GHz}$ as about $2 a=6 \mathrm{Mm}$.

3) The microwave brightness maximum at both frequencies is located near the top of the flaring loop connecting the two HXR brightness maxima.

4) The position of the $34 \mathrm{GHz}$ brightness centre does not coincide with the the $17 \mathrm{GHz}$ brightness centre, and is observed to be situated 2-5" higher than the $17 \mathrm{GHz}$ one (see Fig. 5).

5) The spectral peak at the burst maximum has a very high frequency, about $25 \mathrm{GHz}$.

6) Near the loop footpoints, the spectral slope of the microwave burst is negative, $\alpha<0$, while at the upper part of the loop, near the brightness centre and above, it is positive, $\alpha>0$. In addition, $\alpha$ is negative below the brightness centre of the loop, similar to the regions near footpoints.

7) Subpeaks of the major burst show a very short characteristic decay time, $\leq 2-5 \mathrm{~s}$, at both 17 and $34 \mathrm{GHz}$, and the time delays between microwave and hard X-ray peaks are not resolved in the observation, suggesting that the delays are shorter than one second.

These properties allowed us to conclude that Razin suppression is strong in the central part of the flaring loop. The corresponding model simulations explaining the observed spatial, temporal, and spectral properties show the plasma density and magnetic field inside the loop to be up to $n_{0}=10^{11} \mathrm{~cm}^{-3}$ and $B=50-100 \mathrm{G}$, respectively. X-ray observations confirm the high plasma density in the loop.

Both these values $\left(n_{0}\right.$ and $\left.B\right)$ and the observed properties of pulsations indicate the presence of at least two MHD modes in the loop: the global sausage mode with the period $P_{1}=14-17 \mathrm{~s}$ and the nodes at the footpoints, and another one with the period $P_{2}=8-11 \mathrm{~s}$. The global sausage mode has a maximum magnetic field perturbation at the loop apex and nodes at the footpoints. Indeed, all parts of the loop analysed are observed to oscillate in phase in this mode. This interpretation is in agreement with our previous conclusion (Nakariakov et al. 2003). We exclude the interpretation of the longest period component $\left(P_{1}\right)$ in terms of other MHD modes (kink, ballooning or torsional).

The second periodicity $P_{2}=8-11 \mathrm{~s}$ can be associated with several modes: the second and the third longitudinal harmonics of the kink mode $m=1, l=2$, the third harmonics of the sausage mode and the second harmonics of $m=2, l=2$-ballooning mode. The observed distribution of the oscillation phase along the loop suggests that this mode is likely to have a complicated radial structure, $l>1$. The likely candidates are the modes with $(n=2, m=1, l=2),(n=2$, $m=2, l=2)$ and $(n=3, m=1, l=2)$.

Acknowledgements. The work was partly supported by the RFBR grants No.04-02-39029, 04-02-16753 and by the Royal Society British-Russian Research Collaboration grant. V.M.N. acknowledges the support of a Royal Society Leverhulme Trust Senior Research Fellowship. We acknowledge the MSSL SURF (http://surfwww.mssl.ucl.ac.uk/surf/) for providing SXR data for use in this publication. The authors are grateful to Erwin Verwichte for the help with Fig. 11.

\section{References}

Asai, A., Shimojo, M., Isobe, H., et al. 2001, ApJ, 562, L103

Aschwanden, M. J. 1987, Sol. Phys., 111, 113

Aschwanden, M. J., Fletcher, L., Schrijver, C. J., \& Alexander, D. 1999, ApJ, 520, 880

Dulk, G. A., \& Marsh, K. A. 1982, ApJ, 259, 350

Edwin, P. M., \& Roberts, B. 1982, Sol. Phys., 76, 239

Edwin, P. M., \& Roberts, B. 1983, Sol. Phys., 88, 179

Fleishman, G. D., \& Melnikov, V. F. 2003, ApJ, 587, 823

Grechnev, V. V., White, S. M., \& Kundu, M. R. 2003, ApJ, 588, 1163

Kane, S. R., Kai, K., Kosugi, T., et al. 1983, ApJ, 271, 376

Kiplinger, A. L., Dennis, B. R., Frost, K. J., \& Orwig, L. E. 1983, ApJ, 273, 783

Melnikov, V. F., Shibasaki, K., \& Reznikova, V. E. 2002a, ApJ, 580, L185

Melnikov, V. F, Reznikova, V. E., \& Shibasaki, K. 2002b, Proc. of Intern. Conf. Active processes on the Sun and stars, ed. V. V. Zaitsev, \& L. V. Yasnov, 225

Melnikov, V. F., Reznikova, V. E., Yokoyama, T. \& Shibasaki, K. 2002c, in Solar Variability: From Core to Outer Frontiers (10th European Solar Physics Meeting, 9-14 September 2002, Prague), ed. A. Wilson, ESA SP-506, 339

Melnikov, V. F., Gary, D. E., \& Nita, G. M. 2005, ApJ, in press

Melrose, D. B., \& Brown, J. C. 1976, MNRAS, 176, 15

Nakajima, H., Kosugi, T., Kai, K., \& Enome, S. 1983, Nature, 305, 292

Nakariakov, V. M. 2003, in Dynamic Sun, ed. B. N. Dwivedi, 314

Nakariakov, V. M., Ofman, L., DeLuca, E. E., Roberts, B., \& Davila, J. M. 1999, Science, 285, 862

Nakariakov, V. M., Melnikov, V. F., \& Reznikova, V. E. 2003, A\&A, 412, L7

Nita, G. M., Gary, D. E., \& Lee, J. W. 2004, ApJ, 605, 528

Nobeyama, Radioheliograph Catalog of Events. NRO, Minamimaki, Minamisaku, Nagano 384-1305, Japan, 2002

Ramaty, R., Schwartz, R. A., Enome, S. \& Nakajima, N. 1994, ApJ, 436, 941

Razin, V. A. 1960a, Izv. VUZov Radiofizika, 3, 584

Razin, V. A. 1960b, Izv. VUZov Radiofizika, 3, 921

Rosenberg, H. 1970, A\&A, 9, 159

Sakai, J.-I., \& de Jager, C. 1996, Space Sci. Rev., 77, 1

Shibasaki, K. 2001, ApJ, 557, 326

Twiss, R. Q. 1954, Phyl. Mag., 45, 249

Urpo, S., et al. 1992, Solar Radio Flares 1989-1991, HUT Report 11, Ser. A

Zaitsev, V. V., \& Stepanov, A. V. 1975, Issled. Geomagn. Aeron. Fiz. Solntsa, 37, 3

Zaitsev, V. V., \& Stepanov, A. V. 1982, Sov. Astron. Lett., 8, 132

Zaitsev, V. V., Stepanov, A. V. 1989, Sov. Astron. Lett., 15, 66

Zaitsev, V. V., Stepanov, A. V., Urpo, S., \& Pohjolainen, S. 1998, A\&A, 337, 887

Zaqarashvili, T. V. 2003, A\&A, 399, L15 